\title{
A robust observer of rotor position and speed for IPMSM HFI sensorless drives
}

\author{
$1^{\text {st }}$ Amir MESSALI \\ Centrale Nantes, LS2N UMR \\ CNRS 6004, Nantes, France \\ amir.messali@ec-nantes.fr
}

\author{
$2^{\text {nd }}$ Malek GHANES \\ Centrale Nantes, LS2N UMR \\ CNRS 6004, Nantes, France \\ malek.ghanes@ec-nantes.fr
}

\author{
$3^{\text {rd }}$ Mohamad KOTEICH \\ Renault Group, Technocentre \\ Guyancourt, France \\ $4^{\text {th }}$ Med Assaad HAMIDA \\ Centrale Nantes, LS2N UMR \\ CNRS 6004, Nantes, France \\ mohamad.koteich@renault.com mohamed.hamida@ec-nantes.fr
}

\begin{abstract}
This paper proposes a new tracking algorithm associated to high frequency (HF) signal injection techniques for sensorless control of Interior Permanent Synchronous Machine (IPMSM). The proposed strategy allows to overcome the knowledge of the machine parameters and HF signal characteristics which are the main drawbacks of the existing tracking algorithms (phase-locked loop (PLLs), mechanical system observers, inverse of tangent). Several simulation experimental results are provided to verify and to test the robustness of the proposed sensorless control method in the framework of electric propulsion used in automotive applications.
\end{abstract}

Index Terms-Traching algorithm, Sensorless, sliding mode, IPMSM

\section{INTRODUCTION}

Controlled IPMS motor drives without mechanical speed sensors at the motor shaft have the attractions of low cost and high reliability [1]. In the literature, sensorless control methods for PMSM drives are divided into two main categories according to the operation speed region. The first one is called model based technique these methods [2], [3], [4], [5] are utilized in middle- and high-speed regions [6]. In the lowspeed region, a second category based on high frequency (HF) signal injection methods can be employed [7], [8], [9]. A hybrid position estimation strategy combining the two different methods can achieve whole-speed-range sensorless operation [10].

In the literature of high-frequency injection (HFI) position estimation, there exist several tracking algorithms (PLLs [11], mechanical observers [12], the inverse of tangent [13]). These methods suffer from the knowledge of machine parameters $\left(L_{d}, L_{q}\right.$ inductances, inertia $J$, viscous friction $\left.K_{f}, \ldots\right)$ and the dependency of injected signal characteristics (frequency $\omega_{c}$, magnitude $V_{c}$ ). The present paper proposes a robust solution for rotor position tracking in HFI techniques, using a variable structure observer. The proposed solution has following advantages:

- robustness against machine parameters variations (inductances, inertia,...) and independence from injected signal characteristics (magnitude, frequency).

- operating in all speed ranges, not only at low speed.
- operating with all torque ranges (robustness versus the magnetic saturation).

- possibility to be associated to all HF signal injection methods (especially adaptive frequency signal injection methods).

The robustness and the efficiency of the proposed method are illustrated in case of interior permanent magnet synchronous machine (IPMSM). The pulsating injection method is used in order to introduce the proposed strategy of rotor position/speed estimation (note that others HF injection methods can be also used). Performances of the proposed strategy are highlighted by several simulation and experimental tests.

\section{HF IPMSM MODELS}

The complex notation, $\underline{Z}_{b}^{a}$ is adopted in this paper where the subscript $b$ refers to a stator quantity, whereas the superscript $a$ is either a rotor $(r)$ or stator $(s)$ quantity that allows to know whether the quantity is expressed in the stator $(\alpha \beta)$ or in the rotor $(d q)$ reference frame.

The HF models [14] are obtained by considering following assumptions:

- The HF impedance of the machine is dominated by selfstator inductance $\left(R_{s}<j \omega_{c} L_{s}\right)$, it means that the influence of the stator resistance is neglected.

- In the rotor reference frame, the two axis $(d q)$ being decoupled from each others.

- The rotating Back-EMF is neglected.

Stationary frame voltage model:

$$
v_{s}{ }^{s} \simeq \frac{d \psi_{s}{ }^{s}}{d t} .
$$

Stationary frame flux-current model:

$$
\psi_{\underline{s}}^{s}=L_{0} \underline{i}_{\underline{s}}^{s}+L_{2} i_{\underline{s}}{ }^{*} e^{j 2 \theta} .
$$

From (2), the current expression is deduced

$$
i_{\underline{s}}{ }^{s}=\frac{1}{L_{0}^{2}-L_{2}^{2}}\left(L_{0} \psi_{s}^{s}-L_{2} \psi_{s}^{s *} e^{j 2 \theta}\right) .
$$

where $L_{0}=\frac{L_{d}+L_{q}}{2}$ and $L_{2}=\frac{L_{d}-L_{q}}{2}$ are respectively the average and differential inductances. 


\section{Pulsating injection-BASED MEthod}

\section{A. Injected voltage}

In this part, the HF injected voltage signal [15] is used, in a fixed direction, and added to the $\mathrm{d}$ axis output voltage. The carrier signal in the estimated $(\hat{d} \hat{q})$ frame can be expressed as

$$
v_{s}^{\hat{r}}=-V_{c} \sin \left(\omega_{c} t\right)\left[\begin{array}{l}
1 \\
0
\end{array}\right]
$$

where $V_{c}$ and $\omega_{c}$ are respectively the magnitude and the carrier frequency of the injected signal.

The HF injected voltage in the stator reference frame is expressed

$$
\left[\begin{array}{l}
v_{\alpha} \\
v_{\beta}
\end{array}\right]=-V_{c} \sin \left(\omega_{c} t\right)\left[\begin{array}{c}
\cos (\widehat{\theta}) \\
\sin (\widehat{\theta})
\end{array}\right] .
$$

\section{B. Current resulting from the injected voltage}

The stator flux generated by the high frequency signal injection can be obtained by integrating the injected voltage given in (5) by using (1):

$$
\psi_{s}^{s}=\int-V_{c} \sin \left(\omega_{c} t\right)\left[\begin{array}{l}
\cos (\widehat{\theta}) \\
\sin (\widehat{\theta})
\end{array}\right] d t=\frac{V_{c}}{\omega_{c}} \cos \left(\omega_{c} t\right)\left[\begin{array}{l}
\cos (\widehat{\theta}) \\
\sin (\widehat{\theta})
\end{array}\right]
$$

From (6) and (3), the high frequency stator current is expressed by:

$$
i_{\underline{s}}^{s}=\frac{V_{c}}{\omega_{c}\left(L_{0}^{2}-L_{2}^{2}\right)}\left(L_{0} e^{j \hat{\theta}}-L_{2} e^{j(2 \theta-\hat{\theta})}\right) \cos \left(\omega_{c} t\right)
$$

The general current expression is given by:

$$
i_{\underline{s}}^{s}=I_{c p} \cos \left(\omega_{c} t\right) e^{j \hat{\theta}}-I_{c n} \cos \left(\omega_{c} t\right) e^{j(2 \theta-\hat{\theta})}+i_{s 1}^{s}
$$

where,

$$
\begin{aligned}
I_{c p} & =\frac{L_{0} V_{c}}{\omega_{c}\left(L_{0}^{2}-L_{2}^{2}\right)} \\
I_{c n} & =\frac{L_{2} V_{c}}{\omega_{c}\left(L_{0}^{2}-L_{2}^{2}\right)}
\end{aligned}
$$

and $i_{s 1}^{s}$ are respectively the magnitude of the positive component, the negative component and the fundamental component of the stator current. The stator current (8) is expressed in the estimated frame:

$$
i_{s}^{\hat{r}}=\left[\begin{array}{c}
i_{\hat{d}} \\
i_{\hat{q}}
\end{array}\right]=\left[\begin{array}{c}
I_{c p}-I_{c n} \cos (2(\theta-\hat{\theta})) \\
-I_{c n} \sin (2(\theta-\hat{\theta}))
\end{array}\right] \cos \left(\omega_{c} t\right)+i_{s 1}^{s} e^{-j \hat{\theta}}
$$

\section{Signal processing and error extraction}

Several signal processing techniques have been proposed in the literature [16]. One of these approaches is based on HPF (high pass filter) to remove the fundamental component. Consequently (11) reads

$$
i_{s}^{\hat{r}}=\left[\begin{array}{c}
i_{\hat{d} c} \\
i_{\hat{q} c}
\end{array}\right]=\left[\begin{array}{c}
I_{c p}-I_{c n} \cos 2(\theta-\hat{\theta}) \\
-I_{c n} \sin 2(\theta-\hat{\theta})
\end{array}\right] \cos \left(\omega_{c} t\right)
$$

Then a heterodyning process followed by a LPF (low pass filter) to extract only the position information contained in the current negative sequence is applied. The position error expression can be deduced from the second component of (12) multiplied by $\cos \left(\omega_{c} t\right)$ as follows:

$$
\begin{aligned}
\varepsilon & =\operatorname{LPF}\left(\cos \left(\omega_{c} t\right) i_{\hat{q} c}\right) \\
& =\operatorname{LPF}\left(-I_{c n} \sin 2(\theta-\hat{\theta}) \cos \left(\omega_{c} t\right)^{2}\right) \\
& =\operatorname{LPF}\left(\left(-\frac{I_{c n}}{2} \sin 2(\theta-\hat{\theta})\right)\left(1-\sin \left(\omega_{c} t\right)^{2}\right)\right)
\end{aligned}
$$

Finally, $\varepsilon$ can be expressed as:

$$
\varepsilon=-\frac{I_{c n}}{2} \sin (2(\theta-\hat{\theta})) .
$$

The above developed pulsating method is summarized in Fig.1 where $i_{q}^{r *}$ is the second component of the current reference $i_{s}^{r *}$. In this figure, the block "Robust estimation of $\theta$ and $\omega$ " which represents the main contribution is not developed before, it will be the subject of the next section.

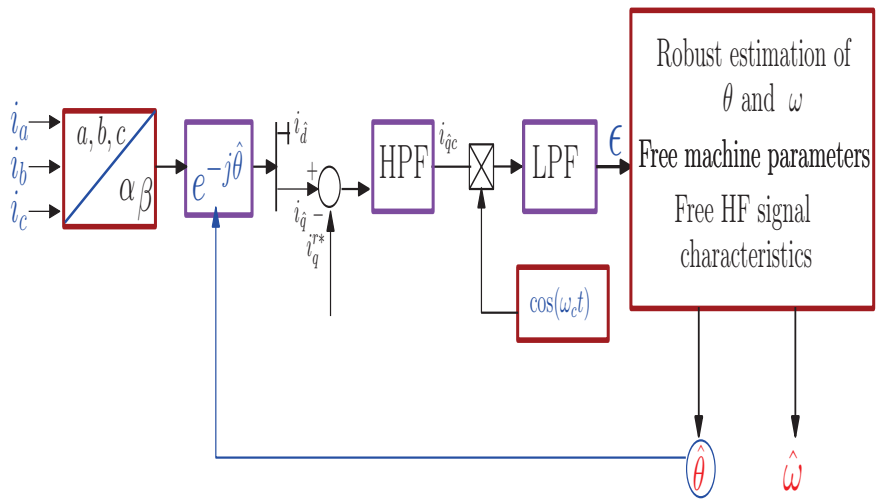

Figure 1. Scheme of rotor position/speed estimation based on pulsating injection technique

\section{PRoposed Robust Rotor POSITION/SPEED ESTIMATION}

\section{A. Motivating idea}

Expression error (14) is linked to the knowledge of the current negative sequence $I_{c n}$ defined in (10). As $I_{c n}$ depends both on machine parameters (inductances $L_{d}, L_{q}$ ) and injected signal characteristics (magnitude $V_{c}$ and frequency $\omega_{c}$ ). All tracking algorithms cited below are not robust to $I_{c n}$ variations for the rotor position/speed estimation of IPM machines in all speed/torque operation ranges.

The novelty of the proposed strategy consists to use only the sign of the position error in the correction term of the estimation algorithm, instead of using the position error $\varepsilon$ defined in (14). The position estimation error $\theta-\hat{\theta}$ is supposed to be small, i.e. $\theta \simeq \hat{\theta}$, (14) becomes

$$
\sigma=\operatorname{sign}\left(-I_{c n} \Delta \theta\right)=\operatorname{sign}\left(-I_{c n}\right) \operatorname{sign}(\Delta \theta)
$$

In general case $-I_{c n}>0$ because $L_{q}>L_{d}$, then (15) can be written as follows

$$
\sigma=\operatorname{sign}(\Delta \theta)
$$

where $\operatorname{sign}(\Delta \theta)$ is the sigmoid function of the form:

$$
\operatorname{sign}(\Delta \theta):\left\{\begin{array}{l}
1 \text { if } \Delta \theta>0 \\
-1 \text { if } \Delta \theta<0 \\
0 \text { if } \Delta \theta=0
\end{array}\right.
$$


and $\Delta \theta=\theta-\hat{\theta}$. It can be argued that equation (15) doesn't depend on machine parameters $\left(L_{d}, L_{q}, J, K_{f}, \ldots\right)$ and injected signal characteristics $\left(V_{c}, \omega_{c}\right)$. Only the sign of the position error is required to estimate the rotor position/speed of IPMSM by the proposed (17)-(18) auto sliding mode observer

$$
\begin{aligned}
\dot{\hat{\omega}} & =K_{\omega} \sigma \\
\dot{\hat{\theta}} & =\hat{\omega}+K_{\theta} \sigma .
\end{aligned}
$$

Assumption 1: The speed variation is assumed to be small with respect to position and electrical quantities in the machine.

From assumption 1, the mechanical double integrator system of position/speed which is used to design the observer (17)(18) is given by:

$$
\begin{aligned}
\dot{\omega} & =0 \\
\dot{\theta} & =\omega
\end{aligned}
$$

\section{B. Stability analysis}

Let be (21)-(22) the position and the speed estimation errors between system (19)-(20) and observer (17)-(18)

$$
\begin{aligned}
& e_{\omega}=\omega-\hat{\omega} \\
& e_{\theta}=\Delta \theta
\end{aligned}
$$

whose dynamics are given by:

$$
\begin{aligned}
& \dot{e}_{\omega}=-K_{\omega} \sigma \\
& \dot{e}_{\theta}=e_{\omega}-K_{\theta} \sigma
\end{aligned}
$$

Theorem 1: Suppose that the assumption 1 holds. Then, the observation algorithm (17)-(18) with parameters $K_{\omega}>0$ and $K_{\theta}>0$ ensures the convergence of estimation position and speed dynamic errors (23)-(24) to zero in a finite-time.

proof: The first step is to analyze the stability of the position estimation error dynamic (24). For that let consider the following candidate Lyapunov function $V_{\theta}$

$$
V_{\theta}=\frac{1}{2} e_{\theta}^{2}
$$

whose derivative reads

$$
\begin{aligned}
\dot{V}_{\theta} & =e_{\theta} \dot{e}_{\theta}=e_{\theta}\left(e_{\omega}-K_{\theta} \operatorname{sign}\left(e_{\theta}\right)\right) \\
& =e_{\theta} e_{\omega}-e_{\theta} K_{\theta} \operatorname{sign}\left(e_{\theta}\right) \leqslant\left|e_{\theta}\right|\left|e_{\omega}\right|-K_{\theta}\left|e_{\theta}\right|
\end{aligned}
$$

Le be

$$
K_{1}=-\left|e_{\omega}\right|+K_{\theta}>0
$$

then (26) can rewritten as

$$
\dot{V}_{\theta} \leq-K_{1}\left|e_{\theta}\right|
$$

which implies that the position estimation error $e_{\theta}$ (22) converges to zero in finite-time $t_{1}>0$ and for all $t \geq t_{1}$, one has

$$
\dot{e}_{\theta}=e_{\theta}=0 \text {. }
$$

Expression (29) means that the sliding condition is reached. Right now, by using condition (29) $\left(\dot{e}_{\theta}=0\right)$ in (24), one has

$$
e_{\omega}=K_{\theta} \operatorname{sign}\left(e_{\theta}\right)
$$

It can be seen from (27) that $K_{\theta}>0$, one can deduce

$$
\operatorname{sign}\left(e_{\omega}\right)=\operatorname{sign}\left(e_{\theta}\right)
$$

Similarly, the stability of the speed estimation error dynamic (23) can be analyzed. Let us define the following candidate Lyapunov function

$$
V_{\omega}=\frac{1}{2} e_{\omega}^{2}
$$

By taking into account (31) in (23), the time derivative of (32) is given by

$$
\dot{V}_{\omega}=e_{\omega} \dot{e}_{\omega}=-K_{\omega} e_{\omega} \operatorname{sign}\left(e_{\omega}\right)
$$

that becomes

$$
\dot{V}_{\omega} \leq-K_{\omega}\left|e_{\omega}\right|
$$

where $K_{\omega}$ is a positive constant. This implies that the error speed estimation $e_{\omega}$ converges to zero in finite time $t_{2}>0$ for all $t \geq t_{2}$.

From (34) and (28), the finite time convergence of the proposed observer (17)-(18) is obtained. This ends the proof.

\section{Tuning}

- Parameters $K_{\omega}>0$ and $K_{\theta}>0$ are chosen according to the stability analysis.

- The injected signal frequency is chosen to be greater than the the nominal machine fundamental frequency and less than the switching inverter frequency (to verify the Shannon property, it should be at most half of the switching frequency).

- The injected signal magnitude is chosen small enough in order to avoid torque ripples and the machine warming.

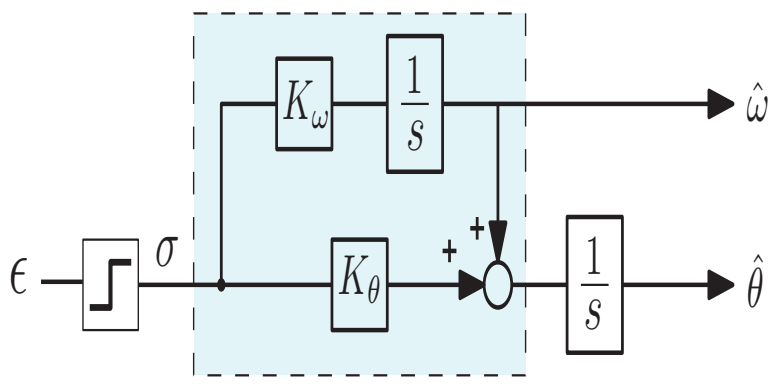

Figure 2. Proposed strategy scheme 


\section{Simulation AND EXPERIMENTAL RESUlts}

Simulations and Experiments were carried out. The sampling period is chosen to $10^{-4} s$ and the PWM frequency is set to $10 \mathrm{kHz}$. The DC voltage is set to $400 \mathrm{~V}$. The motor parameters are given in table I.

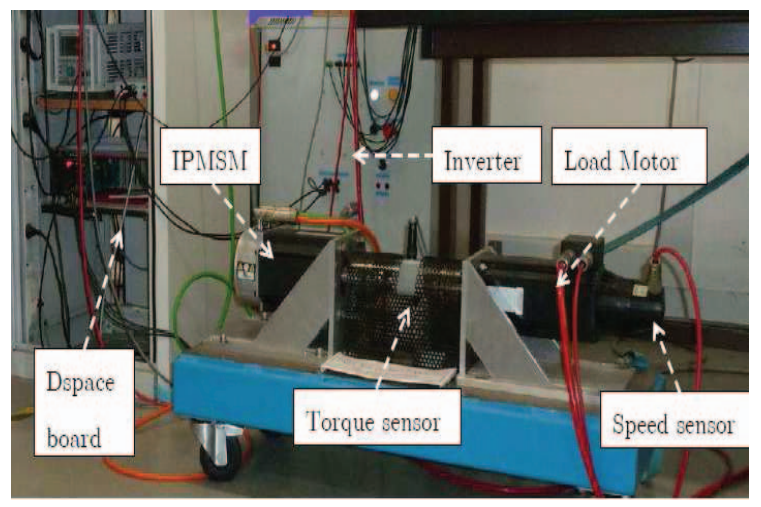

Figure 3. Motor set-up

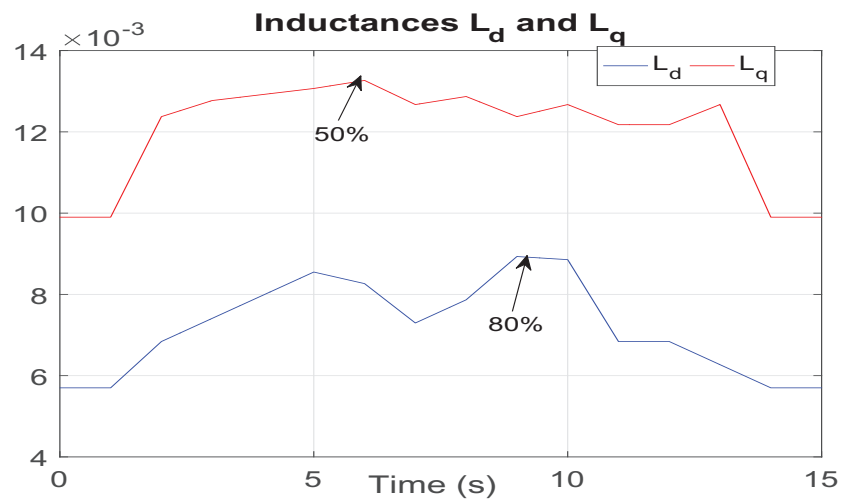

Figure 4. Inductances profiles

The purpose of the used speed benchmark (see the first figure of Fig. 5) is to validate self-sensing control algorithms of the IPMSM in difficult paths. The nominal machine fundamental frequency is $105 \mathrm{~Hz}$. Injected signal characteristics are chosen: $\omega_{c}=1000.2 . \pi(\mathrm{Rad} / \mathrm{s})$ and $V_{c}=4(\mathrm{~V})$. Consequently, the cutoff frequency of the HPF is set at $F_{H P F}=600 \mathrm{~Hz}$. The cutoff frequency of the LPF is set at $F_{L P F}=20 \mathrm{~Hz}$ and the observer parameter values are chosen as follows $k_{\theta}=150$ and $k_{\omega}=1250$. For the simulation, IPMSM inductances are considered very badly known, to prove the insensitivity of the proposed strategy to these variations, the proposed sensorless control strategy is tested under inductances variations. The system performance is tested under $+100 \%$ of the inductances values. To be more close to real-time tests, a white noise is added to current measurements. Simulation and experimental results shown in Fig.6 and Fig.5 display following variables: the measured and the estimated mechanical speeds, the speed estimation error, the measured and the estimated electrical positions, the electrical position estimation error, the reference and the measured current $i_{d}$ and the reference and the measured current $i_{q}$. Notice that, position and speed measurements are only given for comparison with position and speed estimations. From Fig. 5 and Fig. 6, it can be seen that the proposed strategy:

- Gives a good speed estimation. The estimated speed tracks well the measured one, the speed error is centered around zero. However, a small error appears in the acceleration phase which is due to the fact that the speed variation is neglected.

- Gives a good position estimation. The electrical position estimation error is less than \pm 5 degrees. Moreover, the two last plots of Fig. 5 and Fig. 6 display the control stability under nominal steady state mode operation with full torque.

The obtained results confirm the effectiveness and the robustness of the proposed estimation strategy.
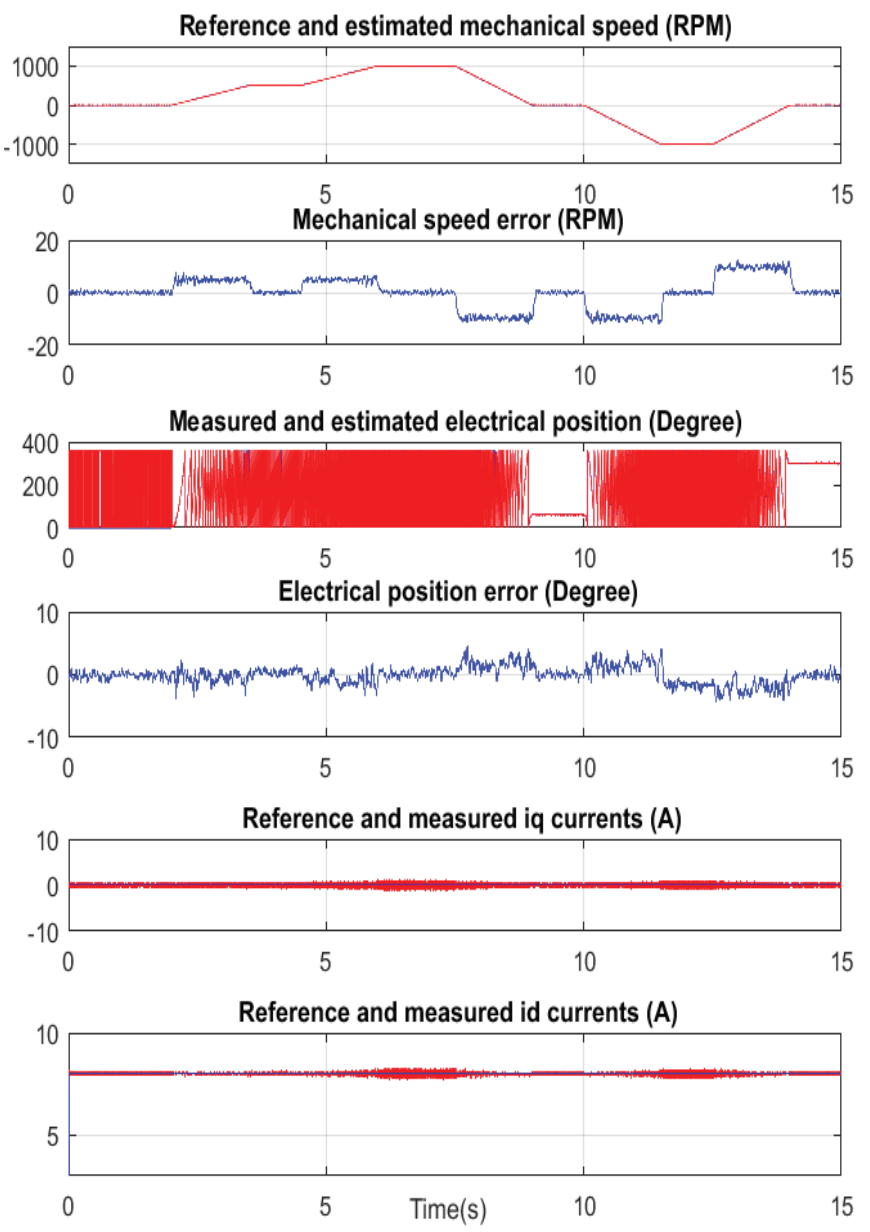

Figure 5. Simulations results 

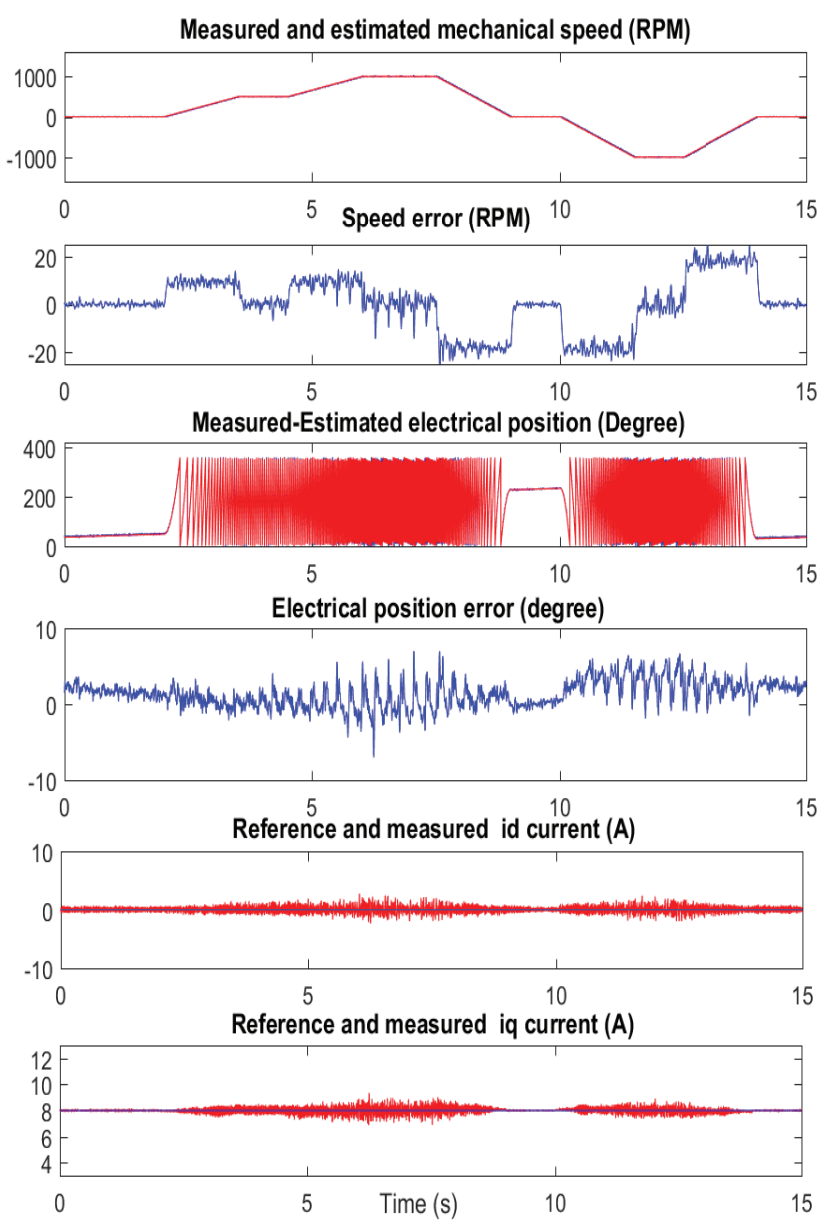

Figure 6. Experimental results

Simulation results are obtained by considering an arbitrary inductances profile shown in Fig. 4, these profiles are introduced to test the robustness of the proposed tracking strategy. The same test is made with the PLL algorithm, once the inductance profiles are considered (see Fig. 4), the PLL algorithm diverges immediately.

\section{CONCLUSION}

In this paper, a robust solution for the rotor position/speed estimation is developed. The proposed strategy is associated to a standard HF signal injection method. Classical techniques used to extract the rotor position/speed information depend on electrical and mechanical machine parameters and injected signal characteristics. The theoretical development, the simulation and the experimental results prove that the proposed strategy does not longer depend on machine parameters and injected signal characteristics, which permit to operate the machine in all speed/torque ranges. These properties allow to the proposed strategy to be a strong candidate to replace PLLs and the mechanical observer widely used in the literature. However, the proposed observer is based on assumption 1. Our future work, that is in progress, will be focused on:

- Design tracking algorithm by taking into account the speed dynamics of the motor.
- Deals with the cross-saturation-phenomenon.

- Reduce the number of filters to reduce the implementation cost and complexity.

- Estimate the phase-shift related to the inverter, high-pass filter and sampling time.

Table I

MOTOR PARAMETERS

\begin{tabular}{|c|c|c|c|}
\hline Speed & $2100 \mathrm{rpm}$ & Torque & $9 \mathrm{Nm}$ \\
\hline$J$ & $0.0073 \mathrm{~kg} . \mathrm{m}^{2}$ & $\Phi_{f}$ & $0.33 \mathrm{~Wb}$ \\
\hline$R_{s}$ & $1.4 \Omega$ & $L_{d}$ & $5.7 \mathrm{mH}$ \\
\hline$p$ & 3 & $L_{q}$ & $9.9 \mathrm{mH}$ \\
\hline
\end{tabular}

\section{ACKNOWLEDGEMENT}

This work was supported by the project Chair between Renault and Centrale Nantes about performances of electric vehicles propulsion.

\section{REFERENCES}

[1] Z. Chen, M. Tomita, S. Doki, and S. Okuma, "An extended electromotive force model for sensorless control of interior permanent-magnet synchronous motors," IEEE Transactions on Industrial Electronics, vol. 50, no. 2, pp. 288-295, Apr. 2003.

[2] M. Hamida, J. de Leon, and A. Glumineau, "Experimental sensorless control for ipmsm by using integral backstepping strategy and adaptive high gain observer," Control Engineering Practice, vol. 59, pp. $64-76,2017$. [Online]. Available: http://www.sciencedirect.com/science/article/pii/S0967066116302611

[3] D. Liang, J. Li, and R. Qu, "Sensorless control of permanent magnet synchronous machine based on second-order sliding-mode observer with online resistance estimation," IEEE Transactions on Industry Applications, vol. 53, no. 4, pp. 3672-3682, July 2017.

[4] G. Zhang, G. Wang, D. Xu, and Y. Yu, "Discrete-time low-frequencyratio synchronous-frame full-order observer for position sensorless ipmsm drives," IEEE Journal of Emerging and Selected Topics in Power Electronics, vol. 5, no. 2, pp. 870-879, June 2017.

[5] M. M. A. Rahman and A. H. M. A. Rahim, "Performance evaluation of ann and anfis based wind speed sensor-less mppt controller," in 2016 5th International Conference on Informatics, Electronics and Vision (ICIEV), May 2016, pp. 542-546.

[6] E. Zerdali and M. Barut, "The comparisons of optimized extended kalman filters for speed-sensorless control of induction motors," IEEE Transactions on Industrial Electronics, vol. 64, no. 6, pp. 4340-4351, June 2017.

[7] N. Bianchi and S. Bolognani, "Influence of rotor geometry of an ipm motor on sensorless control feasibility," IEEE Transactions on Industry Applications, vol. 43, no. 1, pp. 87-96, Jan 2007.

[8] G. Wang, L. Yang, G. Zhang, X. Zhang, and D. Xu, "Comparative investigation of pseudorandom high-frequency signal injection schemes for sensorless ipmsm drives," IEEE Transactions on Power Electronics, vol. 32, no. 3, pp. 2123-2132, 2017.

[9] M. Koteich, A. Messali, and S. Daurelle, "Self-sensing control of the externally-excited synchronous machine for electric vehicle traction application," in 2017 IEEE International Symposium on Sensorless Control for Electrical Drives (SLED), Sept 2017, pp. 91-96.

[10] A. Yousefi-Talouki, P. Pescetto, G. Pellegrino, and I. Boldea, "Combined active flux and high-frequency injection methods for sensorless directflux vector control of synchronous reluctance machines," IEEE Transactions on Power Electronics, vol. 33, no. 3, pp. 2447-2457, March 2018.

[11] L. Harnefors and H. P. Nee, "A general algorithm for speed and position estimation of ac motors," IEEE Transactions on Industrial Electronics, vol. 47, no. 1, pp. 77-83, Feb 2000.

[12] R. D. Lorenz and K. W. V. Patten, "High-resolution velocity estimation for all-digital, ac servo drives," IEEE Transactions on Industry Applications, vol. 27, no. 4, pp. 701-705, Jul 1991. 
[13] S. Medjmadj, D. Diallo, M. Mostefai, C. Delpha, and A. Arias, "Pmsm drive position estimation: Contribution to the high-frequency injection voltage selection issue," IEEE Transactions on Energy Conversion, vol. 30, no. 1, pp. 349-358, March 2015.

[14] G. Wang, L. Yang, G. Zhang, X. Zhang, and D. Xu, "Comparative investigation of pseudorandom high-frequency signal injection schemes for sensorless ipmsm drives," IEEE Transactions on Power Electronics, vol. 32, no. 3, pp. 2123-2132, March 2017.

[15] F. Briz and M. W. Degner, "Rotor position estimation," IEEE Industrial Electronics Magazine, vol. 5, no. 2, pp. 24-36, June 2011.

[16] P. Garcia, F. Briz, M. W. Degner, and D. Diaz-Reigosa, "Accuracy, bandwidth, and stability limits of carrier-signal-injection-based sensorless control methods," IEEE Transactions on Industry Applications, vol. 43, no. 4, pp. 990-1000, July 2007. 